This item was submitted to Loughborough's Research Repository by the author.

Items in Figshare are protected by copyright, with all rights reserved, unless otherwise indicated.

Note on the hydrodynamic description of thin nematic films: strong anchoring model

PLEASE CITE THE PUBLISHED VERSION

http://dx.doi.org/10.1063/1.4816508

PUBLISHER

C) AIP Publishing

VERSION

VoR (Version of Record)

LICENCE

CC BY-NC-ND 4.0

REPOSITORY RECORD

Lin, Te-Sheng, Linda J. Cummings, Andrew J. Archer, Lou Kondic, and Uwe Thiele. 2019. "Note on the Hydrodynamic Description of Thin Nematic Films: Strong Anchoring Model". figshare.

https://hdl.handle.net/2134/16008. 


\section{AIP $\mid$ Physics of Fluids}

\section{Note on the hydrodynamic description of thin nematic films: Strong anchoring model}

Te-Sheng Lin, Linda J. Cummings, Andrew J. Archer, Lou Kondic, and Uwe Thiele

Citation: Physics of Fluids (1994-present) 25, 082102 (2013); doi: 10.1063/1.4816508

View online: http://dx.doi.org/10.1063/1.4816508

View Table of Contents: http://scitation.aip.org/content/aip/journal/pof2/25/8?ver=pdfcov

Published by the AIP Publishing

Articles you may be interested in

Thickness dependence of the anchoring energy of a nematic cell

J. Appl. Phys. 113, 164501 (2013); 10.1063/1.4802677

The isotropic-nematic interface with an oblique anchoring condition

J. Chem. Phys. 131, 174701 (2009); 10.1063/1.3253702

Fingering instabilities in driven thin nematic films

Phys. Fluids 13, 1160 (2001); 10.1063/1.1359748

Anomalous elasticity of disordered nematic gels

AIP Conf. Proc. 519, 214 (2000); 10.1063/1.1291550

Azimuthal anchoring energy of a chiral nematic in cylindrical cavities

Appl. Phys. Lett. 75, 343 (1999); 10.1063/1.124369

\section{AIP $\mid$ chaos CALL FOR APPLICANTS Seeking new Editor-in-Chief}




\title{
Note on the hydrodynamic description of thin nematic films: Strong anchoring model
}

\author{
Te-Sheng Lin, ${ }^{1, a)}$ Linda J. Cummings, ${ }^{2, b)}$ Andrew J. Archer, ${ }^{1, c}$ ) \\ Lou Kondic, ${ }^{2, d)}$ and Uwe Thiele ${ }^{1, e)}$ \\ ${ }^{1}$ Department of Mathematical Sciences, Loughborough University, Loughborough, \\ Leicestershire LE11 3TU, United Kingdom \\ ${ }^{2}$ Department of Mathematical Sciences and Center for Applied Mathematics and Statistics, \\ New Jersey Institute of Technology, Newark, New Jersey 07102, USA
}

(Received 17 January 2013; accepted 8 July 2013; published online 1 August 2013)

\begin{abstract}
We discuss the long-wave hydrodynamic model for a thin film of nematic liquid crystal in the limit of strong anchoring at the free surface and at the substrate. We rigorously clarify how the elastic energy enters the evolution equation for the film thickness in order to provide a solid basis for further investigation: several conflicting models exist in the literature that predict qualitatively different behaviour. We consolidate the various approaches and show that the long-wave model derived through an asymptotic expansion of the full nemato-hydrodynamic equations with consistent boundary conditions agrees with the model one obtains by employing a thermodynamically motivated gradient dynamics formulation based on an underlying free energy functional. As a result, we find that in the case of strong anchoring the elastic distortion energy is always stabilising. To support the discussion in the main part of the paper, an appendix gives the full derivation of the evolution equation for the film thickness via asymptotic expansion. ( 2013 AIP Publishing LLC. [http://dx.doi.org/10.1063/1.4816508]
\end{abstract}

\section{INTRODUCTION}

Thin films of nematic liquid crystals (NLC) have attracted attention over the years, as evidenced by a number of experimental and theoretical studies. ${ }^{1-10}$ When thin nematic films are deposited on solid or liquid substrates, they often exhibit antagonistic anchoring at the free surface and at the substrate, i.e., the director orientation at the substrate is generally parallel to the substrate (planar anchoring) but at the free surface the director is orthogonal to the surface (homeotropic anchoring). As a consequence, the local director orientation changes across the film resulting in an elastic contribution to the energy that should not be neglected: such films are called hybrid films. Sometimes instabilities are observed that result in lateral periodic stripe patterns of the director orientation ${ }^{1-6}$ and film height. However, this is only the case for thin films with thicknesses of several hundred nanometer and below; the wavelength of the stripe patterns diverges at an upper critical film thickness and so, for thicker films, only the usual defects of the nematic phases are observed. ${ }^{3,5}$ Note that spinodal patterns have also been observed, ${ }^{8,11-13}$ normally, in the vicinity of nematic-isotropic or smectic-nematic phase transitions. In contrast, the stripe patterns are observed well inside the nematic region of the liquid crystal phase diagram.

In order to develop a theory for the behaviour of confined nematic liquid crystals, one may calculate the director orientation profile for a given static free surface. Typically, either a flat film or

\footnotetext{
a)Electronic mail: t.lin@lboro.ac.uk

b)Electronic mail: linda.cummings@ njit.edu

c) Electronic mail: a.j.archer@lboro.ac.uk

d) Electronic mail: kondic@njit.edu

e)Electronic mail: u.thiele@ lboro.ac.uk
} 
a periodically deformed state is considered. Such a given static geometry is then used to investigate the director field and to determine its stability. For an imposed flat film, an energy argument allows one to show that there exists a critical thickness ${ }^{14}$

$$
h_{c}=\left|\frac{K}{A_{+}}-\frac{K}{A_{-}}\right|,
$$

where $K$ is the bulk elastic constant of the liquid crystal (in the one constant approximation) and $A_{+}$and $A_{-}$are the anchoring strengths at the free surface and at the substrate, respectively. For thin films, with thickness $h \leq h_{c}$, the director profile is undistorted; the film is in the so-called planar $(\mathrm{P})$ state and the director is aligned parallel to the anchoring angle at the interface with the stronger anchoring strength. For thick films, with film thickness $h>h_{c}$, the state that minimises the free energy is that where the director orientation changes continuously between the two anchoring directions as one moves across the film; this is the Hybrid-Aligned-Nematic (HAN) state introduced above and is the case for the strong anchoring situation considered here. If one assumes that the system is invariant in one direction across the surface on which the film is deposited, so that the film is effectively two-dimensional (2D), one finds that these states are linearly stable. To confirm this assumption, much effort has gone into determining whether the film is laterally stable. ${ }^{1-3,10}$ However, since the film geometry is imposed and static, such analyses cannot account for a possible coupling of variations in film height and director orientation.

In alternative approaches, the long-wave hydrodynamic or so-called lubrication theory has been used successfully in deriving the film thickness evolution equations for films of a variety of different (simple) liquids and to explore the dynamics under the influence of gravity or other body forces, and a variety of surface and interfacial forces. ${ }^{15-21}$ In order to extend this approach to describe films of NLCs, Ben Amar and Cummings ${ }^{7}$ derived a model to describe the surface evolution of NLCs with strong anchoring in 2D settings that was later adapted to model 2D spreading droplets, ${ }^{22}$ spreading droplets with defects ${ }^{23}$ and to account for three-dimensional (3D) settings. ${ }^{24}$ Another long-wave model was introduced by Carou et al. ${ }^{25-27}$ to study blade coating and cavity filling flows of NLC in 2D. However, none of these long-wave evolution equations agree with models that use energy arguments, ${ }^{9}$ when it comes to identifying the effect of the elastic distortion energy on the film dynamics. Antagonistic anchoring is predicted to destabilise the film in Refs. 7 and 22-24, but in Refs. 25-27 it is predicted to have no influence on the stability of the film. In Ref. 9 and 28, however, it is argued on physical grounds that the elastic energy is stabilising. Thus, predictions based on the theory of Refs. 7 and 22-24 are in direct conflict with those from the theory in Refs. 9 and 28.

On a different note, the energetic approach to deriving the long-wave theory mentioned above is based on the fact that, as was noted some time ago, the evolution equation for the height of a thin Newtonian film can be written in a variational form in situations where inertia can be neglected. For nematic liquid crystals, it is not a priori clear whether or not this approach can be applied. In Ref. 9, a model is derived based on an energy argument and a gradient dynamics ansatz that employs a mobility typically for isotropic liquids. However, no mathematical justification was given.

The purpose of this note is to clarify these issues by reconciling the hydrodynamic long-wave and energetic approaches in the case of layers of nematic liquid crystals with strong anchoring, and so to provide a solid basis for further investigations. Our main results are as follows: (1) In the case of strong antagonistic anchoring, the elastic energy contribution always acts so as to stabilise the layer. This is found employing the long-wave approximation of the governing nematohydrodynamic bulk equations with consistent interfacial boundary conditions, and as well by employing a thermodynamically motivated gradient dynamics formulation. (2) The long-wave models of Refs. 7 and 22-24 were derived by employing a stress balance at the free surface of the film as in standard Newtonian flow that is inconsistent with the bulk equations. This leads to a change in sign of the elastic contribution in the film thickness evolution equation. When this boundary condition is modified to also include the elastic stress, results consistent with the energy approach are obtained. (3) The mobility function in the gradient dynamics approach must be obtained from hydrodynamics. Here, we show that the evolution equation for the height of a thin film of nematic liquid crystals derived via asymptotic expansion of the full nematohydrodynamic equations can be written in a variational form and so is consistent with the gradient dynamics approach. 
The paper is organised as follows: The continuum theory of NLC, including the elastic energy and Ericksen-Leslie bulk equations together with consistent boundary conditions, is given in Sec. II. Focusing on the 2D case, the long-wave approximation of the governing equations and boundary conditions is sketched in Sec. III while the full details are given in Appendix A. This allows the reader to easily reproduce our main findings. In Sec. IV, a thermodynamically motivated gradient dynamics formulation is employed to derive the evolution equation of a nematic film. The stability of the free surface is studied through a linear stability analysis. Finally, in Sec. V we compare the results of the two approaches and discuss the validity and limitation of the present model. The note concludes with an outlook on related problems that could be studied based on our results.

\section{CONTINUUM DESCRIPTION OF NEMATIC LIQUID CRYSTAL}

Nematic liquid crystals consist of rod-like molecules that have no positional order, but have long-range orientational order. Thus, the molecules are free to flow as a liquid, but still maintain their long-range directional order. The mean molecule alignment is described by the unit vector $\boldsymbol{n}=\left(n_{1}, n_{2}, n_{3}\right)^{T}$ where the superscript $T$ denotes matrix transposition. Further notation conventions used here are presented in Appendix C.

Distortions of the director field result in a contribution to the free energy, that for NLC is known as the Frank-Oseen elastic energy and reads ${ }^{29,30}$

$$
\begin{aligned}
w_{F}= & \frac{1}{2} K_{1}(\nabla \cdot \boldsymbol{n})^{2}+\frac{1}{2} K_{2}(\boldsymbol{n} \cdot \nabla \times \boldsymbol{n})^{2}+\frac{1}{2} K_{3}(\boldsymbol{n} \times \nabla \times \boldsymbol{n})^{2} \\
& +\frac{1}{2}\left(K_{2}+K_{4}\right) \nabla \cdot((\boldsymbol{n} \cdot \nabla) \boldsymbol{n}-(\nabla \cdot \boldsymbol{n}) \boldsymbol{n}),
\end{aligned}
$$

where $K_{1}, K_{2}$, and $K_{3}$ are the splay, twist, and bend elastic constants, respectively, and $\left(K_{2}+K_{4}\right)$ is called the saddle-splay constant. Note that the saddle-splay term is often omitted since it does not contribute to the governing equations in the case of strong anchoring.

We use the one-constant approximation to simplify the problem. One assumes ${ }^{29,30}$

$$
K \equiv K_{1}=K_{2}=K_{3}, \quad K_{4}=0
$$

and obtains the simplified energy density

$$
w_{F}=\frac{K}{2} \nabla \boldsymbol{n}:(\nabla \boldsymbol{n})^{T}=\frac{K}{2} n_{l, k} n_{l, k}
$$

that enters the nemato-hydrodynamic equations discussed next.

\section{A. Ericksen-Leslie equation}

The bulk flow of NLC may be described by the Ericksen-Leslie equations. ${ }^{29-32}$ The fluid is incompressible, satisfying

$$
\nabla \cdot v=0,
$$

where $\boldsymbol{v}=\left(v_{1}, v_{2}, v_{3}\right)^{T}$ is the velocity field. The momentum balance equation is

$$
\rho \frac{D}{D t} \boldsymbol{v}=\nabla \cdot \boldsymbol{\sigma}
$$

where $\rho$ is the density, $D / D t=\partial / \partial t+\boldsymbol{v} \cdot \nabla$ is the material derivative, $t$ is the time variable, and $\boldsymbol{\sigma}$ is the stress tensor of the NLC. The stress tensor is defined as ${ }^{29}$

$$
\boldsymbol{\sigma}=-p \boldsymbol{I}+\boldsymbol{\sigma}^{E}+\boldsymbol{\sigma}^{V}
$$

where $p$ is the pressure, $\boldsymbol{I}$ is the identity tensor, $\boldsymbol{\sigma}^{E}$ is the elastic (Ericksen) stress tensor, defined by

$$
\boldsymbol{\sigma}^{E}=-\frac{\partial w_{F}}{\partial \nabla \boldsymbol{n}} \cdot(\nabla \boldsymbol{n})^{T}
$$


and $\sigma^{V}$ is the viscous stress tensor with components

$$
\sigma_{i j}^{V}=\alpha_{1} n_{k} n_{p} e_{k p} n_{i} n_{j}+\alpha_{2} N_{i} n_{j}+\alpha_{3} N_{j} n_{i}+\alpha_{4} e_{i j}+\alpha_{5} e_{i k} n_{k} n_{j}+\alpha_{6} e_{j k} n_{k} n_{i},
$$

where

$$
e_{i j}=\frac{1}{2}\left(v_{i, j}+v_{j, i}\right), \quad w_{i j}=\frac{1}{2}\left(v_{i, j}-v_{j, i}\right), \quad N_{i}=\frac{D}{D t} n_{i}-w_{i k} n_{k} .
$$

The $\alpha_{i}$ are constant viscosities.

The equation for the balance of angular momentum is written as (neglecting director inertia)

$$
\nabla \cdot\left(\frac{\partial w_{F}}{\partial \nabla \boldsymbol{n}}\right)-\frac{\partial w_{F}}{\partial \boldsymbol{n}}+\boldsymbol{g}=\lambda \boldsymbol{n},
$$

where the components of $g$ are

$$
g_{i}=-\gamma_{1} N_{i}-\gamma_{2} e_{i, k} n_{k}, \quad \gamma_{1}=\alpha_{3}-\alpha_{2}, \quad \gamma_{2}=\alpha_{6}-\alpha_{5} .
$$

Furthermore, $\lambda$ is the Lagrange multiplier ensuring $|\boldsymbol{n}|=1$.

Under the assumption of the one constant approximation, the Ericksen-Leslie equations, Eqs. (5), (6), and (11), simplify to

$$
\begin{aligned}
\nabla \cdot \boldsymbol{v} & =0, \\
\rho \frac{D}{D t} \boldsymbol{v} & =-\nabla\left(p+w_{F}\right)-K \nabla \boldsymbol{n} \cdot \Delta \boldsymbol{n}+\nabla \cdot \boldsymbol{\sigma}^{V}, \\
K \Delta \boldsymbol{n}+\boldsymbol{g} & =\lambda \boldsymbol{n},
\end{aligned}
$$

respectively, where we have used that

$$
\boldsymbol{\sigma}^{E}=-K \nabla \boldsymbol{n} \cdot(\nabla \boldsymbol{n})^{T},
$$

and

$$
\nabla \cdot\left(\nabla \boldsymbol{n} \cdot(\nabla \boldsymbol{n})^{T}\right)=\frac{1}{2} \nabla\left(\nabla \boldsymbol{n}:(\nabla \boldsymbol{n})^{T}\right)+\nabla \boldsymbol{n} \cdot \Delta \boldsymbol{n} .
$$

As a result, the Ericksen-Leslie equations in the one constant approximation are given by Eq. (13) and need to be solved subject to appropriate boundary conditions.

a. Remark 1: Note that sometimes the stress tensor for NLC is written differently from Eq. (7), e.g., Ref. 33 uses

$$
\tilde{\boldsymbol{\sigma}}=-\left(\tilde{p}+w_{F}\right) \boldsymbol{I}+\boldsymbol{\sigma}^{E}+\boldsymbol{\sigma}^{V} .
$$

However, one may combine the two terms of the isotropic part of $\tilde{\sigma}$ and define a modified pressure as $p=\tilde{p}+w_{F}$. Hence, with the exception of the modified pressure the derivations that follow are not affected.

b. Remark 2: Equation (13b) can be rewritten as

$$
\rho \frac{D}{D t} \boldsymbol{v}=-\nabla\left(p+w_{F}\right)+\nabla \boldsymbol{n} \cdot \boldsymbol{g}+\nabla \cdot \boldsymbol{\sigma}^{V}
$$

by using Eq. (13c) together with $\nabla \boldsymbol{n} \cdot \boldsymbol{n}=\mathbf{0}$. This formulation is more popular in the literature since it only involves the first derivative of the director field.

\section{Boundary conditions}

We assume here that the NLC film sits on a solid substrate at $z=0$ with the free surface (or film thickness) described by $z=h(x, y, t)$. 
For the director field $\boldsymbol{n}$, we impose strong anchoring conditions such that the director is planar at the solid substrate and is homeotropic at the free surface. Specifically, we have

$$
\begin{aligned}
& \boldsymbol{n} \cdot \boldsymbol{z}=0, \quad \text { at } z=0, \\
& \boldsymbol{n} \cdot \boldsymbol{t}_{i}=0, \quad \text { at } z=h(x, y, t),
\end{aligned}
$$

where $z=(0,0,1)^{T}$ and $\boldsymbol{t}_{i}$ are the surface tangent vectors,

$$
\boldsymbol{t}_{\mathbf{1}}=\frac{1}{\sqrt{1+\left(\partial_{x} h\right)^{2}+\left(\partial_{y} h\right)^{2}}}\left(1,0, \partial_{x} h\right)^{T}, \quad \boldsymbol{t}_{\mathbf{2}}=\frac{1}{\sqrt{1+\left(\partial_{x} h\right)^{2}+\left(\partial_{y} h\right)^{2}}}\left(0,1, \partial_{y} h\right)^{T} .
$$

For the velocity field $\boldsymbol{v}$, we assume no-slip and no-penetration at the solid substrate,

$$
v_{1}=v_{2}=v_{3}=0, \quad \text { at } z=0 .
$$

At the free surface, $z=h(x, y, t)$, we have the kinematic condition and balance of normal and tangential stresses. The kinematic condition is

$$
v_{3}=\partial_{t} h+v_{1} \partial_{x} h+v_{2} \partial_{y} h, \quad \text { at } z=h .
$$

For normal stress, we assume that the jump across the interface is balanced by surface tension. That is,

$$
\boldsymbol{k} \cdot\left(\boldsymbol{\sigma}-\boldsymbol{\sigma}^{i}\right) \cdot \boldsymbol{k}=2 \gamma H, \quad \text { at } z=h,
$$

where $\boldsymbol{\sigma}^{i}=-p_{0} \boldsymbol{I}$ is the stress tensor of the air phase, $p_{0}$ is the atmospheric pressure, $\gamma$ is the surface tension, $H$ is the mean curvature, and $\boldsymbol{k}$ is the surface normal vector

$$
\boldsymbol{k}=\frac{1}{\sqrt{1+\left(\partial_{x} h\right)^{2}+\left(\partial_{y} h\right)^{2}}}\left(-\partial_{x} h,-\partial_{y} h, 1\right)^{T} .
$$

For tangential stress, we assume that there is no jump at the interface

$$
\boldsymbol{k} \cdot\left(\sigma-\sigma^{i}\right) \cdot \boldsymbol{t}_{i}=0 .
$$

That is, we assume that no tangential surface tension gradient exists, as is appropriate for strong anchoring. For the case where surface gradient exists, see Ref. 34.

\section{LONG-WAVE HYDRODYNAMIC DESCRIPTION IN TWO DIMENSIONS}

In this section, we restrict attention to two space dimensions and focus on the long-wave approximation of the governing equations presented previously in Sec. II. The full details are given in Appendix A. The aim here is to study the contribution of nematic elasticity to the free surface evolution, and to distinguish results obtained using different scalings and boundary conditions.

Assume the flow is two-dimensional and $y$-independent, so that the director field can be expressed as $\boldsymbol{n}=(\sin \theta, \cos \theta)^{T}$ where the angle $\theta$ is taken as the difference between the director orientation and the positive $z$-axis, as shown in Fig. 1(a), and the velocity field is $v=(u, w)^{T}$. We introduce long-wave scalings to nondimensionalize the governing equations. The scalings are

$$
(x, z)=(L \bar{x}, \delta L \bar{z}), \quad(u, w)=(U \bar{u}, \delta U \bar{w}), \quad t=\frac{L}{U} \bar{t}, \quad p=\frac{\mu U}{\delta^{2} L} \bar{p},
$$

where $U$ is the scale of fluid velocity, $\delta=H / L \ll 1$ is the ratio between the typical film thickness scale, $H$, and a typical lateral length scale, $L$. In addition, in order to focus only on the nematic elasticity, we approximate the nematic viscous stress tensor by its Newtonian equivalent, setting $\sigma_{i j}^{V}=2 \mu e_{i j}$, where $\mu=\alpha_{4} / 2$. 


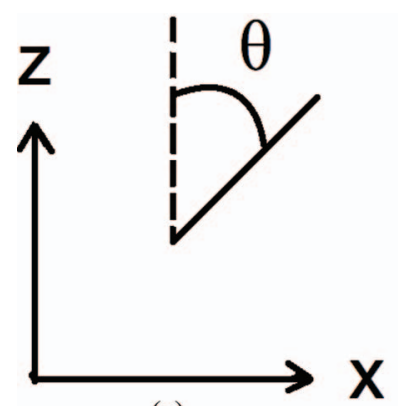

(a)

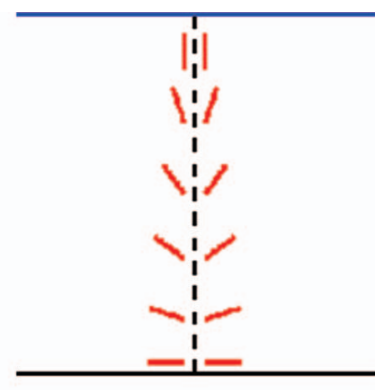

(b)

FIG. 1. The coordinates used in this paper are given in (a). The angle $\theta$ of the director is measured with respect to the positive $z$ axis. In (b), we present two possible director profiles of a hybrid film. The molecules can bend either clockwise or counterclockwise across the film. The solid (blue) top curve indicates the free surface, the solid (black) bottom curve indicates the solid substrate, short (red) lines represent the orientation of the director field, and the dashed (black) line indicates the defect location.

\section{A. Weak elasticity}

Assuming that the elastic free energy is weak compared to the pressure, we can introduce the dimensionless number (inverse Ericksen number)

$$
\bar{K}=\frac{K}{\delta \mu U L} .
$$

The leading order bulk equations are then given by (after dropping the over-bars)

$$
\begin{gathered}
\partial_{x} p=\partial_{z}^{2} u, \\
\partial_{z} p=0, \\
K \partial_{z}^{2} \theta=0, \\
\partial_{x} u+\partial_{z} w=0 .
\end{gathered}
$$

In addition, the leading order boundary conditions are

$$
\begin{gathered}
\theta(z=0)=\frac{\pi}{2}, \quad \theta(z=h)=0, \\
p=p_{0}-C \partial_{x}^{2} h, \quad \partial_{z} u=0, \quad \text { at } z=h,
\end{gathered}
$$

where $C=\delta^{3} \gamma / \mu U$ is the inverse capillary number.

It is easily seen that the velocity field and the director field are decoupled. The film thickness evolution equation is obtained as

$$
\partial_{t} h+\partial_{x}\left(\frac{C}{3} h^{3} \partial_{x}^{3} h\right)=0
$$

and the director field satisfies

$$
\theta=\frac{\pi}{2}\left(1-\frac{z}{h}\right)
$$

This corresponds to the approach taken in Refs. 25-27. 


\section{B. Moderate elasticity}

Instead, if we introduce the inverse Ericksen number as

$$
\bar{K}=\frac{K}{\mu U L},
$$

the leading order bulk equations are given by (after dropping the over-bars)

$$
\begin{aligned}
\partial_{x}\left(p+\frac{K}{2}\left(\partial_{z} \theta\right)^{2}\right) & =\partial_{z}^{2} u, \\
\partial_{z} p & =0, \\
K \partial_{z}^{2} \theta & =0, \\
\partial_{x} u+\partial_{z} w & =0
\end{aligned}
$$

with leading order boundary conditions

$$
\begin{gathered}
\theta(z=0)=\frac{\pi}{2}, \quad \theta(z=h)=0, \\
p=p_{0}-C \partial_{x}^{2} h-K\left(\partial_{z} \theta\right)^{2}, \quad-K\left(\partial_{x} \theta \partial_{z} \theta+\left(\partial_{z} \theta\right)^{2} \partial_{x} h\right)+\partial_{z} u=0, \quad \text { at } z=h .
\end{gathered}
$$

Under such a scaling, the director field is decoupled from the flow and is given by Eq. (29). The tangential stress boundary condition in Eq. (33) is then reduced to $\partial_{z} u(z=h)=0$. We can therefore solve for the pressure and velocity field exactly. As a result, the film evolution equation is given by

$$
\partial_{t} h+\partial_{x}\left(\frac{C}{3} h^{3} \partial_{x}^{3} h-\frac{\widetilde{K}}{3} \partial_{x} h\right)=0
$$

where $\widetilde{K}=\pi^{2} K / 4$.

In contrast, Ben Amar and Cummings ${ }^{7}$ employ Eqs. (31a)-(32) and impose the normal stress balance assuming that the jump of the pressure is balanced by surface tension alone, as is appropriate for Newtonian fluids; that is, they use Eq. (27) instead of Eq. (33). As a result, they obtain an equation much like Eq. (34) but with the opposite sign for the elasticity term. This issue will be discussed later in Sec. V.

\section{GRADIENT DYNAMICS FORMULATION FOR A THIN FILM OF NEMATIC LIQUID CRYSTALS IN TWO DIMENSIONS}

It was noted some time ago that the time evolution equation for the height of a thin Newtonian film on a solid substrate can be written in a variational form in situations where inertia can be neglected. ${ }^{21,35,36}$ The evolution of the film thickness $h$ follows a dissipative gradient dynamics governed by equation

$$
\partial_{t} h=\partial_{x}\left[Q(h) \partial_{x}\left(\frac{\delta F}{\delta h}\right)\right],
$$

where $\delta / \delta h$ denotes functional variation with respect to $h$. The resulting relaxation dynamics is governed by the free energy functional $F$ with the mobility function $Q(h)$.

Such an approach may also be used to obtain the evolution equation for a NLC film in the limit of moderate elasticity discussed above in Sec. III B. Restricting our attention again to a 2D geometry, we simplify the elastic distortion energy for the case of lateral long-wave distortions, i.e., we assume the scalings given in Eq. (23). The bulk elastic energy is to leading order

$$
w_{F}=\frac{K}{2}\left(\partial_{z} \theta\right)^{2}
$$

We further assume that the director adjusts instantaneously to its steady state as compared to the fluid relaxation time, i.e., we assume $K=O(\mu U L)$. Then, the director field can be exactly solved for 
to obtain a linear profile as shown in Eq. (29) assuming strong planar anchoring at the solid substrate and strong homeotropic anchoring at the free surface. The corresponding director orientation across the film is sketched in Fig. 1(b).

As a result, the bulk elastic energy $w_{F}$ (energy/volume) of the NLC can be rewritten as

$$
w_{F}=\frac{\widetilde{K}}{2 h^{2}} .
$$

The free energy functional is then expressed as

$$
F=\int C d s+\int\left(\int_{0}^{h} w_{F} d z\right) d x \approx \int\left[C\left(1+\frac{\left(\partial_{x} h\right)^{2}}{2}\right)+\frac{\widetilde{K}}{2 h}\right] d x,
$$

where $d s \approx\left(1+\left(\partial_{x} h\right)^{2} / 2\right) d x$ is the approximated surface element. The evolution equation of the film is given in gradient dynamics formulation by introducing $F$ into Eq. (35)

$$
h_{t}=-\partial_{x}\left[Q(h) \partial_{x}\left(C \partial_{x}^{2} h+\frac{\widetilde{K}}{2 h^{2}}\right)\right],
$$

where the mobility function $Q(h)$ can be obtained from the Poiseuille NLC flow, Eq. (A25). One should note that Eqs. (39) and (34) are identical when $Q(h)=h^{3} / 3$.

\section{A. Linear stability analysis}

To have a basic understanding of the elastic contribution to the stability of NLC free surface, we analyse the linear stability of a flat film, $h=h_{0}$. Assuming $h=h_{0}+\xi$, $\xi \ll h_{0}$ in Eq. (39), to leading order we have

$$
\partial_{t} \xi=-Q\left(h_{0}\right)\left(C \partial_{x}^{4} \xi-\frac{\tilde{K}}{h_{0}^{3}} \partial_{x}^{2} \xi\right)
$$

With the harmonic mode ansatz $\xi=\exp (i k x+\omega t)$, one obtains the dispersion relation

$$
\omega=-Q\left(h_{0}\right)\left(C k^{4}+\frac{\widetilde{K}}{h_{0}^{3}} k^{2}\right) .
$$

Note that the constants $C, \tilde{K}$ and the film height $h_{0}$ are always positive and therefore the growth rate $\omega$ is negative for any wavenumber $k$. This implies that the elastic term is always stabilising and in the case of strong anchoring the flat film $h=h_{0}$ is always stable if only capillarity and elasticity are taken into account.

\section{DISCUSSION AND CONCLUSION}

We have consolidated several approaches to derive the evolution equation for free surface films of nematic liquid crystals with strong anchoring at both interfaces. We have demonstrated the consistency between the long-wave approximation model, Eqs. (34) and (A24), and the model derived through a thermodynamically motivated gradient dynamics formulation, Eq. (39). The elastic energy contribution acts in a stabilising manner in each of these models, consistent with the physically motivated arguments of, e.g., Refs. 9 and 28.

In contrast, the long-wave models of Refs. 7 and 22-24, which use an alternative normal stress balance that is not consistent with the bulk equations, lead to qualitatively different results. The normal stress boundary condition in these papers neglects the contribution of the elastic stress tensor, which leads to a change in sign of the elastic contribution in the free surface evolution equation. A third approach used by Carou et al. ${ }^{25-27}$ scales the nematic elasticity such that, to leading order, the free surface is unaffected by the elasticity, and one recovers the Newtonian thin film equation, Eq. (28).

One should note that the strong anchoring models presented here are only valid for rather thick films as noted in Sec. I. First, the main assumption of the model - the strong anchoring of the 
director at both interfaces - is only valid for $h \gg h_{c}$ where $h_{c}$ is defined in Eq. (1). For moderate film thickness $h \approx h_{c}$ or even thinner, the surface anchoring energy has to be taken into account (see Refs. 37 and 38 for the interfacial boundary conditions of static NLC). This may be done via an $a d$ hoc amendment of the free surface anchoring condition in Eq. (32) (cf. the approach taken in Ref. 22, for the model with the "Newtonian" normal stress balance). Alternatively, it may be modelled via the variational approach which will be the subject of future work.

Second, as for isotropic liquids with film thickness below about $100 \mathrm{~nm}$, long- and short-range effective intermolecular forces between the substrate and the free surface have to be taken into account possibly through a Derjaguin or disjoining pressure that describes wettability effects. ${ }^{39}$ For nematic liquid crystals, the influence of van der Waals interactions has been discussed, e.g., in Refs. 8 and 40. Additional Casimir-type forces may be induced by fluctuations of the director orientation, most notably in very thin films with uniform director orientation, i.e., in the planar $(\mathrm{P})$ state. ${ }^{40,41}$ Note, however, that the notions "disjoining pressure" or "structural disjoining pressure" are used in Refs. 42-44 to denote the pressure contribution resulting from the elasticity of the liquid crystal, i.e., the last term in Eq. (39).

We would also like to point out that, within the present long-wave scalings (Eq. (23)), there is no distinction in the elastic energy whether the director is bent clockwise or counterclockwise. The two director profiles shown in Fig. 1(b) (on the right hand side and on the left hand side of the dashed (black) line) have exactly the same elastic energy, $\widetilde{K} / 2 h^{2}$. However, such a situation is still not allowed even though the elastic energy is continuous across the dashed (black) line. The director field is discontinuous and it breaks the long-wave assumption $\left(\partial_{x}^{2} \theta \ll \partial_{z}^{2} \theta\right)$. A simple way to circumvent this was proposed in Ref. 23, whereby the discontinuity of Fig. 1(b) is smoothed out over a given range. More sophisticated models for real defects are needed. For instance, one may incorporate a description of the dynamics of the scalar order parameter related to the nematic-isotropic transition. Away from the phase transition, it can be employed to model defects. Such a model would also allow one to tackle the structuring of films that occurs close to the nematic-isotropic transition. ${ }^{8,11-13}$

In conclusion, we have clarified how the elastic contribution influences the free surface of a nematic film under the strong anchoring assumptions. Within the long-wave scalings, we have discussed two cases, corresponding to weak and moderate elasticity, respectively:

- $\frac{K}{\mu U L}=O(\delta)$ : The bulk elasticity has only a minor influence on the free surface evolution. It does not affect the stability of a film. The evolution of the film and the director field are given by Eqs. (A18) and (A20), respectively.

- $\frac{K}{\mu U L}=O(1)$ : The strong antagonistic anchoring makes a significant contribution leading to a diffusion-like term in the film surface height evolution equation - see Eq. (39). Furthermore, the director always maintains its steady state, given by Eq. (29).

The models can be derived either from asymptotic expansion of the nemato-hydrodynamic equations or from a thermodynamically motivated gradient dynamics formulation. The former approach has the advantage of mathematical rigorousness, while the latter approach is much simpler in deriving the evolution equations. It is found that the elastic distortion energy is always stabilising.

\section{ACKNOWLEDGMENTS}

T.L. and U.T. acknowledge support from the European Union (EU) under Grant No. MRTNCT-2004-005728 (MULTIFLOW). U.T. would like to thank Günter Grün for an invitation to Bonn in October 2004 where they did a derivation similar to that in the appendix and discussed the sign of the elasticity term in the resulting evolution equation. L.J.C. and L.K. acknowledge support from the National Science Foundation (NSF) under Award Nos. DMS-0908158 and DMS-1211713, and L.J.C. also acknowledges support from KAUST under Award No. KUK-C1-013-04. 


\section{APPENDIX A: LONG WAVELENGTH APPROXIMATION OF A THIN FILM OF NLC}

\section{Ericksen-Leslie equations in two spatial dimensions}

Assume the flow is two-dimensional and $y$-independent, then the director field can be expressed as $\boldsymbol{n}=(\sin \theta, \cos \theta)^{T}$ and the velocity field is $\boldsymbol{v}=(u, w)^{T}$. The elastic energy reduces to

$$
w_{F}=\frac{K}{2}\left(\left(\partial_{x} \theta\right)^{2}+\left(\partial_{z} \theta\right)^{2}\right) .
$$

Without fluid inertia, the linear momentum equations are then given by (from Eq. (13b))

$$
\begin{aligned}
& \partial_{x}\left[p+\frac{K}{2}\left(\left(\partial_{x} \theta\right)^{2}+\left(\partial_{z} \theta\right)^{2}\right)\right]=-K \partial_{x} \theta\left(\partial_{x}^{2} \theta+\partial_{z}^{2} \theta\right)+\partial_{x} \sigma_{11}^{V}+\partial_{z} \sigma_{12}^{V}, \\
& \partial_{z}\left[p+\frac{K}{2}\left(\left(\partial_{x} \theta\right)^{2}+\left(\partial_{z} \theta\right)^{2}\right)\right]=-K \partial_{z} \theta\left(\partial_{x}^{2} \theta+\partial_{z}^{2} \theta\right)+\partial_{x} \sigma_{21}^{V}+\partial_{z} \sigma_{22}^{V} .
\end{aligned}
$$

(The viscous stress tensor, $\sigma^{V}$, is defined in Appendix B, Eq. (B1).) For the angular momentum equation, Eq. (13c), one can eliminate the Lagrange multiplier $\lambda$ by performing an inner product with the vector $\boldsymbol{n}^{\perp}=(\cos \theta,-\sin \theta)^{T}$. We then have

$$
\begin{aligned}
K\left(\partial_{x}^{2} \theta+\partial_{z}^{2} \theta\right)= & \gamma_{1}\left[\dot{\theta}-\frac{1}{2}\left(\partial_{z} u-\partial_{x} w\right)\right] \\
& +\frac{\gamma_{2}}{2}\left[\left(\partial_{x} u-\partial_{z} w\right) \sin (2 \theta)+\left(\partial_{z} u+\partial_{x} w\right) \cos (2 \theta)\right] .
\end{aligned}
$$

The continuity equation, Eq. (13a), is rewritten as

$$
\partial_{x} u+\partial_{z} w=0 .
$$

\section{a. Boundary conditions}

In 2D, the boundary conditions for the director field, assuming strong anchoring at both interfaces (planar at the substrate and homeotropic at the free surface), are

$$
\theta(z=0)=\frac{\pi}{2}, \quad \theta(z=h)=\cos ^{-1}\left(\frac{1}{\sqrt{1+\left(\partial_{x} h\right)^{2}}}\right) .
$$

For the velocity field, we assume no-slip at the solid substrate,

$$
u=w=0, \quad \text { at } \quad z=0 .
$$

At the free surface, we have the kinematic boundary condition

$$
w=\partial_{t} h+u \partial_{x} h, \quad \text { at } \quad z=h,
$$

which can be combined with the incompressibility condition, Eq. (A5), to be

$$
\partial_{t} h+\partial_{x}\left(\int_{0}^{h} u d z\right)=0
$$

or equivalently,

$$
\partial_{t} h+\partial_{x}\left(\int_{0}^{h} \partial_{z} u(h-z) d z\right)=0 .
$$

(Note that the no-slip boundary condition, $u(z=0)=0$ was imposed in deriving Eq. (A10).)

For the balance of normal and tangential stresses, we first note that the stress tensor for a NLC film is written as

$$
\boldsymbol{\sigma}=-p\left[\begin{array}{cc}
1 & 0 \\
0 & 1
\end{array}\right]-K\left[\begin{array}{cc}
\left(\partial_{x} \theta\right)^{2} & \partial_{x} \theta \partial_{z} \theta \\
\partial_{x} \theta \partial_{z} \theta & \left(\partial_{z} \theta\right)^{2}
\end{array}\right]+\left[\begin{array}{cc}
\sigma_{11}^{V} & \sigma_{12}^{V} \\
\sigma_{21}^{V} & \sigma_{22}^{V}
\end{array}\right]
$$


and the stress tensor of the air phase is $\sigma^{i}=-p_{0} \boldsymbol{I}$. We assume that the jump in the normal stress is balanced by surface tension and the jump in tangential stress is zero. That is,

$$
\boldsymbol{k} \cdot\left(\sigma-\sigma^{i}\right) \cdot \boldsymbol{k}=\gamma \kappa, \quad \boldsymbol{k} \cdot\left(\sigma-\sigma^{i}\right) \cdot \boldsymbol{t}=0,
$$

where $\kappa$ is the curvature, $\boldsymbol{k}$ is the normal vector at the free surface, and $\boldsymbol{t}$ is the tangent vector at the free surface, defined as

$$
\kappa=\frac{\partial_{x}^{2} h}{\left(1+\left(\partial_{x} h\right)^{2}\right)^{3 / 2}}, \quad \boldsymbol{k}=\frac{1}{\sqrt{1+\left(\partial_{x} h\right)^{2}}}\left(-\partial_{x} h, 1\right)^{T}, \quad \boldsymbol{t}=\frac{1}{\sqrt{1+\left(\partial_{x} h\right)^{2}}}\left(1, \partial_{x} h\right)^{T},
$$

respectively.

\section{Non-dimensionalisation and long-wave approximation}

We make the usual long-wave scalings to nondimensionalize the governing equations as shown in Eq. (23). Also we rescale the coefficients of nematic viscosity by the Newtonian equivalent, setting $\alpha_{i}=\mu \bar{\alpha}_{i}$ where $\mu=\alpha_{4} / 2$. For the elastic constant, we assume $K=\epsilon \mu U L \bar{K}$ where $\epsilon$ is a parameter of order $o(1 / \delta)$ that will be specified later.

The leading order equations are then given by (after dropping the over-bars)

$$
\begin{aligned}
\partial_{x}\left(p+\frac{\epsilon K}{2}\left(\partial_{z} \theta\right)^{2}\right) & =-\epsilon K \partial_{x} \theta \partial_{z}^{2} \theta+\partial_{z}\left(q_{1}(\theta) \partial_{z} u\right) \\
\partial_{z}\left(p+\frac{\epsilon K}{2}\left(\partial_{z} \theta\right)^{2}\right) & =-\epsilon K \partial_{z} \theta \partial_{z}^{2} \theta \\
\epsilon K \partial_{z}^{2} \theta & =-\delta q_{2}(\theta) \partial_{z} u \\
\partial_{x} u+\partial_{z} w & =0
\end{aligned}
$$

where $q_{1}(\theta)$ and $q_{2}(\theta)$ are related to the viscous stress tensor, their full expressions are given later in Appendix A 2 b. The leading order boundary conditions are the kinematic boundary condition, Eq. (A10), with

$$
\begin{gathered}
\theta(z=0)=\frac{\pi}{2}, \quad \theta(z=h)=0, \\
p=p_{0}-C \partial_{x}^{2} h-\epsilon K\left(\partial_{z} \theta\right)^{2}, \quad-\epsilon K\left(\partial_{x} \theta \partial_{z} \theta+\left(\partial_{z} \theta\right)^{2} \partial_{x} h\right)+q_{1}(\theta) \partial_{z} u=0, \quad \text { at } z=h,
\end{gathered}
$$

where $C=\delta^{3} \gamma / \mu U$ is the inverse capillary number.

\section{a. Weak elasticity $(\epsilon=\delta)$}

Assuming the elastic free energy is weak compared to the pressure, we can choose $\epsilon=\delta$. Observing that Eq. (A14b) reduces to $p_{z}=0$ at leading order, one can solve the pressure exactly and the velocity is then determined by

$$
\partial_{z} u(x, z)=\frac{C}{q_{1}(\theta)}(h-z) \partial_{x}^{3} h .
$$

Hence, by using Eq. (A10), we obtain the film evolution equation as

$$
h_{t}+C \partial_{x}\left(Q(h) \partial_{x}^{3} h\right)=0,
$$

where

$$
Q(h)=\int_{0}^{h} \frac{(h-z)^{2}}{q_{1}(\theta)} d z
$$


In addition, the director field satisfies

$$
\partial_{z}^{2} \theta=\frac{C}{K} \frac{q_{2}(\theta)}{q_{1}(\theta)}(z-h) \partial_{x}^{3} h
$$

with boundary conditions defined in Eq. (A15).

One can see that the nematic elasticity as well as viscosity only have influence on the mobility function $Q$, and thus have no influence on the stability of a free surface. This formulation has been studied extensively by Carou et al. ${ }^{25-27}$ both analytically and numerically under the assumption of small director variation.

\section{b. Moderate elasticity $(\epsilon=1)$}

On the other hand, if we have $\epsilon=1$, Eq. (A14c) reduces to $\partial_{z}^{2} \theta=0$ at leading order and hence the director field reaches a linear profile in $z$ as shown in Eq. (29). Moreover, Eqs. (A14a) and (A14b) are simplified to

$$
\begin{aligned}
\partial_{x}\left(p+\frac{\widetilde{K}}{2 h^{2}}\right) & =\partial_{z}\left(q_{1}(\theta) \partial_{z} u\right), \\
\partial_{z} p & =0,
\end{aligned}
$$

with boundary conditions at the free surface

$$
p=p_{0}-C \partial_{x}^{2} h-\frac{\widetilde{K}}{h^{2}}, \quad \partial_{z} u=0 .
$$

We can therefore solve the pressure and velocity field as

$$
p(x, z)=p_{0}-C \partial_{x}^{2} h-\frac{\widetilde{K}}{h^{2}}, \quad \partial_{z} u(x, z)=\left(-C \partial_{x}^{3} h+\frac{\widetilde{K}}{h^{3}} \partial_{x} h\right)\left(\frac{z-h}{q_{1}(\theta)}\right) .
$$

As a result, the film evolution equation is given by

$$
h_{t}+\partial_{x}\left[Q(h)\left(C \partial_{x}^{3} h-\frac{\widetilde{K}}{h^{3}} \partial_{x} h\right)\right]=0,
$$

where $Q(h)$ can be evaluated explicitly as ${ }^{7}$

$$
Q(h)=Q_{0} h^{3}, \quad Q_{0}=\left(\frac{2}{\pi}\right)^{3} \int_{0}^{\pi / 2} \frac{\xi^{2}}{q_{1}(\xi)} d \xi .
$$

\section{APPENDIX B: VISCOUS STRESS TENSOR OF NEMATIC LIQUID CRYSTAL IN TWO DIMENSION}

The viscous stress tensor of NLC in $2 \mathrm{D}$ is written as

$$
\begin{aligned}
\boldsymbol{\sigma}^{V}= & \alpha_{1}\left(\sin ^{2} \theta \partial_{x} u+\cos ^{2} \theta \partial_{z} w+\sin \theta \cos \theta\left(\partial_{z} u+\partial_{x} w\right)\right)\left[\begin{array}{cc}
\sin ^{2} \theta & \sin \theta \cos \theta \\
\sin \theta \cos \theta & \cos ^{2} \theta
\end{array}\right] \\
& +\alpha_{2}\left(\frac{D}{D t} \theta-\frac{\partial_{z} u-\partial_{x} w}{2}\right)\left[\begin{array}{cc}
\sin \theta \cos \theta & \cos ^{2} \theta \\
-\sin ^{2} \theta & -\sin \theta \cos \theta
\end{array}\right] \\
& +\alpha_{3}\left(\frac{D}{D t} \theta-\frac{\partial_{z} u-\partial_{x} w}{2}\right)\left[\begin{array}{cc}
\sin \theta \cos \theta & -\sin ^{2} \theta \\
-\cos ^{2} \theta & -\sin \theta \cos \theta
\end{array}\right] \\
& +\frac{\alpha_{4}}{2}\left[\begin{array}{cc}
2 \partial_{x} u & \partial_{z} u+\partial_{x} w \\
\partial_{z} u+\partial_{x} w & 2 \partial_{z} w
\end{array}\right]
\end{aligned}
$$




$$
\begin{aligned}
& +\frac{\alpha_{5}}{2}\left[\begin{array}{cc}
2 \sin ^{2} \theta \partial_{x} u+\sin \theta \cos \theta\left(\partial_{z} u+\partial_{x} w\right) & 2 \sin \theta \cos \theta \partial_{x} u+\cos ^{2} \theta\left(\partial_{z} u+\partial_{x} w\right) \\
\sin ^{2} \theta\left(\partial_{z} u+\partial_{x} w\right)+2 \sin \theta \cos \theta \partial_{z} w & \sin \theta \cos \theta\left(\partial_{z} u+\partial_{x} w\right)+2 \cos ^{2} \theta \partial_{z} w
\end{array}\right] \\
& +\frac{\alpha_{6}}{2}\left[\begin{array}{cc}
2 \sin ^{2} \theta \partial_{x} u+\sin \theta \cos \theta\left(\partial_{z} u+\partial_{x} w\right) & \sin ^{2} \theta\left(\partial_{z} u+\partial_{x} w\right)+2 \sin \theta \cos \theta \partial_{z} w \\
\cos ^{2} \theta\left(\partial_{z} u+\partial_{x} w\right)+2 \sin \theta \cos \theta \partial_{x} u & \sin \theta \cos \theta\left(\partial_{z} u+\partial_{x} w\right)+2 \cos ^{2} \theta \partial_{z} w
\end{array}\right] .
\end{aligned}
$$

Similarly, the coupling term, $\boldsymbol{g}$, between the director and velocity field can be written as

$$
\boldsymbol{g}=-\gamma_{1}\left(\dot{\theta}-\frac{\partial_{z} u-\partial_{x} w}{2}\right)\left[\begin{array}{c}
\cos \theta \\
-\sin \theta
\end{array}\right]-\frac{\gamma_{2}}{2}\left[\begin{array}{c}
2 \sin \theta \partial_{x} u+\left(\partial_{z} u+\partial_{x} w\right) \cos \theta \\
\left(\partial_{z} u+\partial_{x} w\right) \sin \theta+2 \cos \theta \partial_{z} w
\end{array}\right] .
$$

We also note that, within the long-wave scalings [Eq. (23)], to leading order we have

$$
\sigma_{12}^{V}=\mu q_{1}(\theta) \partial_{z} u+O\left(\frac{\mu U}{L}\right), \quad \boldsymbol{n}^{\perp} \cdot \boldsymbol{g}=-\mu q_{2}(\theta) \partial_{z} u+O\left(\frac{\mu U}{L}\right),
$$

where $\mu=\alpha_{4} / 2, \boldsymbol{n}^{\perp}=(\cos \theta,-\sin \theta)^{T}$ and

$$
\begin{aligned}
& \mu q_{1}(\theta)=\frac{1}{2}\left[\alpha_{4}+2 \alpha_{1} \sin ^{2} \theta \cos ^{2} \theta+\left(\alpha_{5}-\alpha_{2}\right) \cos ^{2} \theta+\left(\alpha_{3}+\alpha_{6}\right) \sin ^{2} \theta\right], \\
& \mu q_{2}(\theta)=\frac{1}{2}\left[\gamma_{1}-\gamma_{2} \cos (2 \theta)\right] .
\end{aligned}
$$

As an example, for Newtonian fluids, $q_{1}(\theta)=1$ and $q_{2}(\theta)=0$.

\section{APPENDIX C: NOTATION CONVENTIONS}

For clarity, we list all the notations used. We write for a vector $\boldsymbol{n}=n_{i}$ or $\boldsymbol{m}=m_{i}$, for a tensor $\boldsymbol{\sigma}=\sigma_{i j}$ or $\boldsymbol{\kappa}=\kappa_{i j}$; as the superscript $T$ denotes transposition one has $\boldsymbol{\sigma}^{T}=\sigma_{j i}$. Further, $\epsilon_{i j k}$ is the alternator. The notations for operators and products are $\nabla \boldsymbol{n}=n_{j, i}, \nabla \cdot \boldsymbol{n}=n_{k, k}, \nabla \times \boldsymbol{n}=\epsilon_{i l k} n_{k, l}$, $\Delta \boldsymbol{n}=n_{i, k k}, \nabla \cdot \boldsymbol{\sigma}=\sigma_{i k, k}, \boldsymbol{\sigma} \cdot \boldsymbol{\kappa}=\sigma_{i k} \kappa_{k j}, \boldsymbol{\sigma}: \boldsymbol{\kappa}=\sigma_{k l} \kappa_{l k}, \boldsymbol{\sigma} \cdot \boldsymbol{n}=\sigma_{i k} n_{k}, \boldsymbol{n} \times \boldsymbol{m}=\epsilon_{i l k} n_{k} m_{l}$, where " $i$ " denotes the partial derivative with respect to the $i$ th component.

${ }^{1}$ O. D. Lavrentovich and V. M. Pergamenshchik, "Stripe domain phase of a thin nematic film and the K13 divergence term," Phys. Rev. Lett. 73, 979 (1994).

${ }^{2}$ A. Sparavigna, O. D. Lavrentovich, and A. Strigazzi, "Periodic stripe domains and hybrid-alignment regime in nematic liquid crystals: Threshold analysis," Phys. Rev. E 49, 1344 (1994).

${ }^{3}$ O. D. Lavrentovich and V. M. Pergamenshchik, "Patterns in thin liquid crystal films and divergence elasticity," Int. J. Mod. Phys. B 9, 2389 (1995).

${ }^{4}$ U. Delabre, C. Richard, G. Guéna, J. Meunier, and A. M. Cazabat, "Nematic pancakes revisited,” Langmuir 24, 3998 (2008).

${ }^{5}$ U. Delabre, C. Richard, Y. Sang, and A. M. Cazabat, "Thin liquid crystal films on liquids in the nematic range of temperatures," Langmuir 26, 13368 (2010).

${ }^{6}$ A. M. Cazabat, U. Delabre, C. Richard, and Y. Y. Cheung Sang, "Experimental study of hybrid nematic wetting films," Adv. Colloid Interface Sci. 168, 29 (2011).

${ }^{7}$ M. Ben Amar and L. J. Cummings, "Fingering instabilities in driven thin nematic films," Phys. Fluids 13, 1160 (2001).

${ }^{8}$ D. Van Effenterre, M. P. Valignat, and D. Roux, "Coupling between the nematic/isotropic transition and a thickness transition: A theoretical approach," Europhys. Lett. 62, 526 (2003).

${ }^{9}$ S. Mechkov, A. M. Cazabat, and G. Oshanin, "Post-Tanner spreading of nematic droplets," J. Phys.: Condens. Matter 21, 464134 (2009).

${ }^{10}$ O. V. Manyuhina, A. M. Cazabat, and M. Ben Amar, "Instability patterns in ultrathin nematic films: Comparison between theory and experiment," Europhys. Lett. 92, 16005 (2010).

${ }^{11}$ S. Herminghaus, K. Jacobs, K. Mecke, J. Bischof, A. Fery, M. Ibn-Elhaj, and S. Schlagowski, "Spinodal dewetting in liquid crystal and liquid metal films," Science 282, 916 (1998).

${ }^{12}$ S. Schlagowski, K. Jacobs, and S. Herminghaus, "Nucleation-induced undulative instability in thin films of nCB liquid crystals," Europhys. Lett. 57, 519 (2002).

${ }^{13}$ R. Garcia, E. Subashi, and M. Fukuto, "Thin-thick coexistence behavior of $8 \mathrm{CB}$ liquid crystalline films on silicon," Phys. Rev. Lett. 100, 197801 (2008).

${ }^{14}$ G. Barbero and R. Berberi, "Critical thickness of a hybrid aligned nematic liquid crystal cell," J. Phys. (Paris) 44, 609 (1983). 
${ }^{15}$ A. Oron, S. H. Davis, and S. G. Bankoff, "Long-scale evolution of thin liquid films," Rev. Mod. Phys. 69, 931 (1997).

${ }^{16}$ K. J. Ruschak, "Coating flow,” Annu. Rev. Fluid Mech. 17, 65 (1985).

${ }^{17}$ H. C. Chang and E. A. Demekhin, Complex Wave Dynamics on Thin Films (Elsevier, New York, 2002).

${ }^{18}$ L. Kondic, "Instabilities in gravity driven flow of thin fluid films," SIAM Rev. 45, 95 (2003).

${ }^{19}$ H. A. Stone, A. D. Stroock, and A. Ajdari, "Engineering flows in small devices," Annu. Rev. Fluid Mech. 36, 381 (2004).

${ }^{20}$ R. V. Craster and O. K. Matar, "Dynamics and stability of thin liquid films," Rev. Mod. Phys. 81, 1131 (2009).

${ }^{21}$ U. Thiele, "Thin film evolution equations from (evaporating) dewetting liquid layers to epitaxial growth," J. Phys.: Condens. Matter 22, 084019 (2010).

${ }^{22}$ L. J. Cummings, T.-S. Lin, and L. Kondic, "Modeling and simulations of the spreading and destabilization of nematic droplets," Phys. Fluids 23, 043102 (2011).

${ }^{23}$ T.-S. Lin, L. Kondic, and L. J. Cummings, "Defect modeling in spreading nematic droplets," Phys. Rev. E 85, 012702 (2012).

${ }^{24}$ L. J. Cummings, "Evolution of a thin film of nematic liquid crystal with anisotropic surface energy," Eur. J. Appl. Math. 15, 651 (2004)

${ }^{25}$ J. Q. Carou, B. R. Duffy, N. J. Mottram, and S. K. Wilson, "Steady flow of a nematic liquid crystal in a slowly varying channel," Mol. Cryst. Liq. Cryst. 438, 237 (2005).

${ }^{26}$ J. Q. Carou, B. R. Duffy, N. J. Mottram, and S. K. Wilson, "Shear-driven and pressure-driven flow of a nematic liquid crystal in a slowly varying channel," Phys. Fluids 18, 027105 (2006).

${ }^{27}$ J. Q. Carou, N. J. Mottram, S. K. Wilson, and B. R. Duffy, "A mathematical model for blade coating of a nematic liquid crystal," Liq. Cryst. 34, 621 (2007).

${ }^{28}$ F. Vandenbrouck, M. P. Valignat, and A. M. Cazabat, "Thin nematic films: Metastability and spinodal dewetting," Phys. Rev. Lett. 82, 2693 (1999).

${ }^{29}$ P. G. De Gennes and J. Prost, The Physics of Liquid Crystals (Oxford University Press, New York, 1995).

${ }^{30}$ I. W. Stewart, The Static and Dynamic Continuum Theory of Liquid Crystals (CRC Press, London, 2004).

${ }^{31}$ J. L. Ericksen, "Anisotropic fluids," Arch. Ration. Mech. Anal. 4, 231 (1959).

${ }^{32}$ F. M. Leslie, "Some constitutive equations for liquid crystals," Arch. Ration. Mech. Anal. 28, 265 (1968).

${ }^{33}$ A. D. Rey, "Young-Laplace equation for liquid crystal interfaces," J. Chem. Phys. 113, 10820 (2000).

${ }^{34}$ A. D. Rey, "Nemato-capillarity theory and the orientation-induced Marangoni flow," Liq. Cryst. 26, 913 (1999).

${ }^{35}$ A. Oron and P. Rosenau, "Formation of patterns induced by thermocapillarity and gravity," J. Phys. (France) 2, 131 (1992).

${ }^{36}$ V. Mitlin, "Dewetting of solid surface: Analogy with spinodal decomposition," J. Colloid Interface Sci. 156, 491 (1993).

${ }^{37}$ P. J. Barratt and J. T. Jenkins, "Interfacial effects in the magnetohydrostatic theory of nematic liquid crystals," J. Phys. A: Math. Nucl. Gen. 6, 756 (1973).

${ }^{38}$ J. T. Jenkins and P. J. Barratt, "Interfacial effects in the static theory of nematic liquid crystals," Q. J. Mech. Appl. Math. 27, 111 (1974).

${ }^{39}$ V. M. Starov and M. G. Velarde, "Surface forces and wetting phenomena" J. Phys.: Condens. Matter 21, 464121 (2009).

${ }^{40} \mathrm{P}$. Ziherl and S. Zumer, "Morphology and structure of thin liquid-crystalline films at nematic-isotropic transition," Eur. Phys. J. E 12, 361 (2003).

${ }^{41}$ P. Ziherl, R. Podgornik, and S. Zumer, "Pseudo-Casimir structural force drives spinodal dewetting in nematic liquid crystals," Phys. Rev. Lett. 84, 1228 (2000).

${ }^{42}$ E. Perez, J. E. Proust, L. Ter-Minassian-Saraga, and E. Manev, "Structural disjoining pressure in thin film of liquid crystals I: Thermodynamics and Frederiksz transition with surface fields," Colloid Polym. Sci. 255, 1003 (1977).

${ }^{43}$ A. D. Rey, "Mechanical theory for nematic thin films," Langmuir 17, 1922 (2001).

${ }^{44}$ A. D. Rey, "Capillary models for liquid crystal fibers, membranes, films, and drops," Soft Matter 3, 1349 (2007). 